\title{
Table of EU directives and regulations
}

\section{DIRECTIVES}

Consumer Guarantees Directive 1999/44

268

Art. 7(2)

317

Credit Institutions Directive 2000/12

441

Defective Products Directive

$85 / 374$

Art. 2

Art. 6

360

Art. 7(e)

360

Art. 11

361

Art. 15

361

361

Defective Products Directive 1999/34

360

Distance Contracts Directive $97 / 7$

268

Art. 12(2)

317

Distance Marketing of Services

Directive 2002/65

Art. 12(2)

268

317

Electronic Commerce Directive 2000/31

93, 336

Art. 2(c)

94, 100, 285

Financial Collateral Arrangements Directive 2002/47

441

Insurance Directive

73/239

121

Art. 5(d)

121

Insurance Directive $88 / 357$
$115,121,266$, $268,312,324$, 325-8
Art. 2(d)

324

Art. 5

121, 325

Art. 7

323, 325, 327

Art. 7(1)

328

Art. 7(1)(a)-(d) $\mathbf{3 2 5}$

Art. 7(1)(a) $\mathbf{3 2 5}$

Art. 7(1)(b) $\quad 326$

Art. 7(1)(c) 326

Art. 7(1)(d) $\quad 326$

Art. 7(1)(e) $\quad \mathbf{3 2 6}$

Art. 7(1)(f) 323, 324, 325

Art. 7(1)(g) 325

Art. 7(1)(h) $\quad 325,326$

Art. 7(1)(i) 265, 327

Art. 7(2) $\quad 328$

Art. 7(2)(i) $\quad 327$

Art. 7(2)(ii) $\quad 327$

Art. 7(2)(iii) $\quad 327$

Art. 7(3) $\quad 328$

Art. 8(4)(c) 327

Insurance Directive 90/618

121

Art. 2

121

Insurance Directive 92/49

Art. 27

323, 324

Life Insurance Directive

90/619

$115,266,268$, 312, 328-9

Art. 2(e) $\quad 324$

Art. $4 \quad 323,328$

Art. 4(3) 265, 327

Posting of Workers Directive

96/71

269, 322

Art. 1(1)

322

Art. 1(3) 
$\begin{array}{ll}\text { Art. 2(1) } & \mathbf{3 2 2} \\ \text { Art. 3 } & \mathbf{3 2 3} \\ \text { Art. 6 } & \mathbf{4 0 , 1 3 1}\end{array}$

Protection of Employees on Insolvency Directive 80/987

454

Reorganization of Credit Institutions

Directive 2001/24

441

Self-Employed Commercial Agents Directive 86/653

Art. 17

269, 309

Art. 18

269, 309

Settlement Finality Directive 98/26

441, 461

Television Broadcasting Directive $89 / 552$

368

Art. 2(3)

368

Television Broadcasting Directive 90/618

Art. 2

325

Timeshare Directive 94/47

268

Art. 9

317

Unfair Terms in Consumer Contracts

Directive 93/13 $\mathbf{1 2 8}$

Art. 2(c)

312

Art. 6(2)

316, 317,

318

\section{REGULATIONS}

Brussels I Regulation

$$
\begin{array}{ll}
44 / 2001 \quad 6,8,13,15,16, \\
18,43,46,60, \\
152,154,228, \\
250,251,254, \\
258,267,317, \\
319,331,384, \\
385,398,400, \\
407,425,441, \\
460
\end{array}
$$

Chapter II

(Arts 2-31) 19, 20, 34, 37,

$38,42,45$, 47-9, 107, 113, $154,176,186$, 198, 203, 206, 218, 219, 222, 223, 253, 432, 433

Chapter III

(Arts 32-56)

19, 20, 34, 35, 39, 40, 42, 111, 150, 154, 189, 205, 206, 208-11, 218, 221-4, 230, 238, 239, 434, 435, 450

Chapter IV (Arts 57-58) 21, 43, 205, 206, 236, 239, 433

Chapter V (Arts 59-65) 21

Chapter VI

(Art. 66) 21

Chapter VII (Arts 67-72) 21

Chapter VIII (Arts 73-76) 21

Art. 1

18, 37, 178, 198, 203, 205, 215, 238, 243, 251

Art. 1(1)

21, 22, 431

Art. 1(2)

21, 24, 35

Art. 1(2)(a) 25-8, 69, 103, 225, 384, 430

Art. 1(2)(b) 28, 29, 178, 439

Art. 1(2)(c) $\quad 30$

Art. 1(2)(d) 119, 194, 207 


\begin{tabular}{|c|c|c|c|}
\hline Art. 1(3) & $13,45,205$ & Art. 5(2) & $25-7,47,86$, \\
\hline Arts $2-4$ & $19,45,66,169$ & & $89,384,385$, \\
\hline Arts $2-7$ & 155 & & $430,432,433$ \\
\hline \multirow[t]{4}{*}{ Art. 2} & 19, 45, 47, & Arts 5(3)-(5) & 148 \\
\hline & $\begin{array}{l}49-53,56,59 \\
64,67,76,86 \\
94,96,98,104 \\
114,140,143\end{array}$ & Art. 5(3) & $\begin{array}{l}56,64,67,68, \\
71,86-94, \\
96-9,106,117, \\
143,335,349\end{array}$ \\
\hline & $148,169,172$, & Art. 5(4) & 24,99 \\
\hline & $\begin{array}{l}\text { 173, 182, 194, } \\
198,432\end{array}$ & Art. 5(5) & $\begin{array}{l}64,86,94,99 \\
101,114,116\end{array}$ \\
\hline Art. 2(2) & 48 & & $118,127,128$, \\
\hline \multirow[t]{2}{*}{ Art. 3} & $\begin{array}{l}19,20,45,54 \\
55,59,64,104\end{array}$ & & $\begin{array}{l}131,285,320, \\
451\end{array}$ \\
\hline & 182,393 & Art. 5(6) & $89,102,103$ \\
\hline Art. 3(1) & $45,55,56$ & Art. 5(7) & 89,103 \\
\hline Art. 3(2) & $45,55,56,221$ & Art. 6 & $19,56,57,65$, \\
\hline \multirow[t]{3}{*}{ Art. 4} & $45,51,57,59$, & & $106,169,190$ \\
\hline & $\begin{array}{l}67,110,113, \\
117,128,148, \\
169,171-3, \\
191,219,221,\end{array}$ & Art. 6(1) & $\begin{array}{l}56,64,67, \\
107-9,111, \\
117,143,144, \\
149,188\end{array}$ \\
\hline & $222,393,442$ & Art. 6(2) & 37, 67, 109, \\
\hline Art. 4(2) & 57 & & $110,111,118$ \\
\hline Arts 5-7 & $19,64,66,67$ & & 167,169 \\
\hline Arts 5-24 & $55,194,198$ & Art. 6(3) & $111,112,114$ \\
\hline \multirow[t]{2}{*}{ Art. 5} & $19,56,57,64$, & & $127,131,164$ \\
\hline & $65,67,169$ & Art. 6(4) & 112,136 \\
\hline \multirow[t]{4}{*}{ Art. 5(1) } & $\begin{array}{l}18,47,50,56, \\
64,67,69-74,\end{array}$ & Art. 7 & $\begin{array}{l}19,64-6,104 \\
105\end{array}$ \\
\hline & $\begin{array}{l}\text { 76, 78, 79, } \\
81-9,101,106\end{array}$ & Arts 8-14 & $\begin{array}{l}19,48,113-15, \\
119,124,219\end{array}$ \\
\hline & $\begin{array}{l}110,117,128 \\
131,140,168\end{array}$ & Art. 8 & $\begin{array}{l}\text { 69, 113, 114, } \\
116-18\end{array}$ \\
\hline & 265 & Art. 9 & 18 \\
\hline \multirow[t]{2}{*}{ Art. 5(1)(a) } & $69,70,74,75$ & Art. 9(1)(a) & $47,114,116$ \\
\hline & $78,80,81,84$ & Art. 9(1)(b) & $47,116,117$ \\
\hline \multirow[t]{2}{*}{ Art. 5(1)(b) } & 69, 70, 74, & Art. 9(1)(c) & 117 \\
\hline & $78-85$ & Art. 9(2) & $58,99,113,116$ \\
\hline Art. 5(1)(b)(ii) & 79 & Art. 10 & 117 \\
\hline \multirow[t]{2}{*}{ Art. 5(1)(c) } & $70,74,78,80$, & Art. 11(1) & 118 \\
\hline & 81,84 & Art. 11(2) & 116,117 \\
\hline
\end{tabular}




\begin{tabular}{|c|c|c|c|}
\hline Art. 11(3) & 118 & Art. 18(1) & $113,114,128$, \\
\hline Art. 12 & 50 & & 131 \\
\hline Art. 12(1) & $47,114,118$ & Art. 18(2) & $58,99,114,129$ \\
\hline Art. 12(2) & $114,118,119$ & Art. 19(1) & $47,114,129$ \\
\hline \multirow[t]{3}{*}{ Art. 13} & $20,119,127$ & Art. 19(2) & 129,131 \\
\hline & $132,155,156$, & Art. 19(2)(a) & 129 \\
\hline & 165 & Art. 19(2)(b) & 129, 131 \\
\hline \multirow[t]{2}{*}{ Art. 13(1) } & $118,119,124$, & Art. 20 & 132 \\
\hline & 165 & Art. 20(1) & $47,114,131$ \\
\hline Art. 13(2) & 118,119 & Art. 20(2) & $114,131,132$ \\
\hline Art. 13(3) & $119,120,124$ & Art. 21 & $20,34,132$ \\
\hline Art. 13(4) & 120 & & $155,156,165$, \\
\hline Art. 13(5) & 115,120 & & 186 \\
\hline Art. 14 & $18,20,120,155$ & Art. 21(1) & 132,165 \\
\hline Art. 14(1)-(4) & 120 & Art. 21(2) & 131 \\
\hline Art. 14(3) & 121 & Art. 22 & $20,45,48,53$, \\
\hline Art. 14(4) & 120,121 & & $54,57,68,102$ \\
\hline Art. 14(5) & 115,121 & & $133,134,136$, \\
\hline Arts $15-17$ & $\begin{array}{l}19,70,113, \\
122-4,128, \\
219\end{array}$ & & $\begin{array}{l}142,143,155 \\
156,165,166, \\
174,185-7,\end{array}$ \\
\hline Art. 15 & $\begin{array}{l}69,70,122, \\
126,312\end{array}$ & & $\begin{array}{l}190,198,218, \\
219,237\end{array}$ \\
\hline Art. 15(1) & $\begin{array}{l}113,114,122 \\
124,127\end{array}$ & Art. 22(1) & $\begin{array}{l}18,68,69,103, \\
112,133-9,\end{array}$ \\
\hline Art. 15(1)(c) & $124-6,314$ & & 142,187 \\
\hline Art. 15(2) & $\begin{array}{l}58,99,100 \\
113,126\end{array}$ & Art. 22(2) & $\begin{array}{l}63,133 \\
140-42,187\end{array}$ \\
\hline Art. 15(3) & 124 & & 237 \\
\hline Art. 16 & 47 & Art. 22(3) & 135,142 \\
\hline Art. 16(1) & $\begin{array}{l}47,114,126, \\
127\end{array}$ & Art. 22(4) & $\begin{array}{l}97,133,142-5, \\
219\end{array}$ \\
\hline Art. 16(2) & 114,127 & Art. 22(5) & 154 \\
\hline Art. 16(3) & 114,127 & Art. 23 & $18,19,35,39$, \\
\hline Art. 17 & $\begin{array}{l}20,127,132, \\
155,156,165\end{array}$ & & $\begin{array}{l}45,48,50,53 \\
54,56,57,81\end{array}$ \\
\hline Art. 17(1) & $127,128,165$ & & $82,85,86,104$ \\
\hline Art. 17(2) & 127,128 & & $106,118,119$, \\
\hline Art. 17(3) & 128 & & $127,131,136$, \\
\hline Arts 18-21 & $\begin{array}{l}18,19,113, \\
128,129,219\end{array}$ & & $\begin{array}{l}140,149,152 \\
155,156,158\end{array}$ \\
\hline Art. 18 & 69 & & 161-3, 166-74, \\
\hline
\end{tabular}


186, 191, 194,

198, 267, 433

Art. 23(1)

$54,155,156$,

$164,168,169$,

171

Art. 23(1)(a) $\quad 164$

Art. 23(2) 155, 157

Art. 23(3) 155, 168, 171

Art. 23(4)

Art. 23(5)

Art. 24

Art. 25

102, 103, 155

$54,133,155$,

165,166

20, 50, 56, 58,

$110,118,127$,

131-3, 149,

152,155 ,

172-5, 433

20, 67, 133,

144, 145, 218,

395

Arts 26-31

Art. 26

Art. 26(1)

Art. 26(2)

Art. 26(3)

Art. 26(4)

Arts 27-30

Art. 27

Art. 27(1)

Art. 27(2)

Art. 28
114, 395

39, 55, 230, 231

20, 39, 54, 55,

67, 68, 218,

395

20, 55, 230,

246, 395

20, 55, 230,

246

20, 55, 230, 246

20, 33, 37, 48,

53, 54, 106,

118, 127, 131,

145, 149, 176,

178, 179, 183

39, 48, 58, 105 ,

176, 179-83,

185-8, 191,

193, 194, 218,

235, 236, 435

198

198

39, 48, 58, 105 ,
106, 176-8,

180-82,

187-92, 235

Art. 28(1)

189-91

Art. 28(2)

189, 190

Art. 28(3)

107, 111, 164, 180, 187, 188,

191

Art. 29

Art. 30

41, 58, 136,

177, 187

18, 47, 177,

$183,184,396$

Art. 30(1) $\quad \mathbf{1 8 3}$

Art. 30(2) $\quad 183$

Art. 31

20, 32, 55, 118 ,

127, 131, 150,

175, 198-201,

203, 222, 223,

397,433

Arts 32-56 $\mathbf{2 0 5}$

Art. 32

37, 206, 207,

252

Arts 33-35 37

Art. 33

145, 206,

213

Art. 33(1) 211

Art. 33(2) 211, 239

Art. 33(3) 211, 239

Arts 34-36 210, 244

Art. $34 \quad \mathbf{1 8}, \mathbf{3 0}, \mathbf{2 1 3}$,

214, 230, 235,

242

Art. 34(1) 216, 217, 224, 226, 234, 237, 448

Art. 34(2) 209, 224, 226, 229-33, 399,

427

Art. 34(3) 188, 224, 234-7, 434

Art. 34(4)

229, 234, 236, 237, 434 


\begin{tabular}{|c|c|c|c|}
\hline \multirow[t]{3}{*}{ Art. 35} & $34,113,133$ & Art. 46(2) & 246 \\
\hline & $185,213,214$ & Art. 46(3) & 247 \\
\hline & 242,442 & Art. 47 & $242-4,249$ \\
\hline Art. 35(1) & $145,195,219$ & Art. 47(1) & 238,244 \\
\hline Art. 35(2) & 219,220 & Art. 47(2) & 243 \\
\hline Art. 35(3) & $213,217,224$ & Art. 47(3) & 243 \\
\hline Art. 36 & $\begin{array}{l}213,215,216 \\
224\end{array}$ & Art. 48 & $\begin{array}{l}27,227,238, \\
431\end{array}$ \\
\hline \multirow[t]{2}{*}{ Art. 37} & $190,208,214$, & Art. 49 & 214,215 \\
\hline & $246,247,397$ & Art. 50 & 242 \\
\hline Arts $38-56$ & $210,211,238$ & Art. 51 & 241 \\
\hline \multirow[t]{3}{*}{ Art. 38} & 37, 206, 208, & Arts 53-55 & $18,214,241$ \\
\hline & $210,213,214$ & Art. 53 & 242,246 \\
\hline & 244,250 & Art. 53(1) & 241 \\
\hline Art. 38(1) & 239 & Art. 53(2) & 209,241 \\
\hline Art. 38(2) & 238,240 & Art. 54 & 241 \\
\hline Art. 39(1) & 239 & Art. 55(1) & 241 \\
\hline Art. 39(2) & 240 & Art. 55(2) & 241 \\
\hline Art. 40 & 240 & Art. 56 & 241,242 \\
\hline Art. 40(1) & 240 & Arts $57-58$ & 205,211 \\
\hline Art. 40(2) & 240 & Art. 57 & 212 \\
\hline Art. 40(3) & 241 & Art. 57(2) & 212 \\
\hline Art. 41 & 242,244 & Art. 57(4) & 241 \\
\hline Art. 42(1) & 243 & Art. 58 & 212,241 \\
\hline Art. 42(2) & 241,243 & Art. 59 & $21,58,59,152$ \\
\hline \multirow[t]{2}{*}{ Art. 43} & $243,246,247$ & & 432 \\
\hline & 249 & Art. 60 & $18,21,62,63$, \\
\hline Art. 43(1) & 243,244 & & 140,220 \\
\hline Art. 43(3) & 244 & Art. 60(1) & 58,62 \\
\hline Art. 43(4) & 245 & Art. $60(1)(b)$ & 62 \\
\hline Art. 43(5) & 243,245 & Art. $60(1)(\mathrm{c})$ & 63 \\
\hline Art. 44 & 248,249 & Art. 60(2) & 62 \\
\hline \multirow[t]{2}{*}{ Art. 45} & $133,185,210$, & Art. 60(3) & 102 \\
\hline & 244 & Art. 61 & $24,99,226,234$ \\
\hline \multirow[t]{3}{*}{ Art. 45(1) } & $213,214,217$ & Art. 62 & 208,252 \\
\hline & $229,230,234$, & Art. 63 & 69, 82, 164 \\
\hline & 244,248 & Art. 64 & 105 \\
\hline \multirow[t]{2}{*}{ Art. 45(2) } & $213,215,216$ & Art. 65 & 111,118 \\
\hline & 224,247 & Art. 66 & $21,42,206$ \\
\hline \multirow[t]{2}{*}{ Art. 46} & $18,208,214$, & & 221 \\
\hline & 246-9 & Art. 66(1) & $41-3,206$ \\
\hline Art. 46(1) & 246,249 & Art. 66(2)(a) & 42,206 \\
\hline
\end{tabular}




\begin{tabular}{|c|c|c|c|}
\hline Art. $66(2)(b)$ & \multirow{2}{*}{$\begin{array}{l}42,206,221, \\
388\end{array}$} & Art. 12 & 411 \\
\hline & & Art. 13(1) & 424 \\
\hline Art. 67 & $29,40,148$ & Art. 13(2) & 425 \\
\hline Art. 68 & 59 & Art. 13(3) & 425 \\
\hline Art. 68(1) & 14,205 & Art. 14 & 425 \\
\hline Art. 68(2) & $14,16,450$ & Arts $15-20$ & 426 \\
\hline Art. 69 & $42,206,222$ & Art. 15(2) & 426 \\
\hline Art. 70(2) & 42,206 & Art. 16 & 398 \\
\hline Art. 71 & $18,31,34$ & Art. 37 & 408 \\
\hline & 37-9, 103, 104, & Art. 41 & 392 \\
\hline & $119,146,155$ & Art. 42 & 407 \\
\hline & 179, 205, 216, & Art. 42(1) & 407 \\
\hline & 222 & Art. 42(2) & 389 \\
\hline Art. 71(1) & 38,39 & Art. 46 & 387 \\
\hline Art. 71(2)(a) & 38,39 & Brussels IIA Re & tion \\
\hline Art. 71(2)(b) & 39 & $2201 / 2003$ & $8,9,25,383$ \\
\hline Art. 72 & $18,59,219-21$ & & $384,385,399$, \\
\hline Annex II & 13 & & $400,403-5$ \\
\hline Annex III & 13 & & $409,433,435$ \\
\hline russels II Regu & & Chapter II & 387, 388, 391, \\
\hline $1347 / 2000$ & $\begin{array}{l}8,9,25,383 \\
384,385,386\end{array}$ & & $\begin{array}{l}395,407,408, \\
412\end{array}$ \\
\hline & $399,400,403$ & Chapter III & 387, 389, 390, \\
\hline Chapter II & $387,388,407$ & & 397, 407, 408, \\
\hline Chapter III & $387,388,389$, & & $422-5$ \\
\hline & $397,424,425$ & Art. 1(1) & 386 \\
\hline Art. 1(1)(a) & 385 & Art. 1(1)(a) & 397 \\
\hline Art. 1(1)(b) & 385,424 & Art. 1(1)(b) & 403,405 \\
\hline Art. 1(3) & 383 & Art. 1(2) & 406 \\
\hline Art. 1(3)(e) & 385 & Art. 1(3) & 406 \\
\hline Art. 2 & 391,410 & Art. 1(3)(e) & 430 \\
\hline Art. 3 & 410 & Art. 2 & 386 \\
\hline Art. 3(1) & 410 & Art. 2(1) & 386,406 \\
\hline Art. 3(2) & 411,414 & Art. 2(2) & 386,406 \\
\hline Art. 3(3) & 411 & Art. 2(3) & 383,403 \\
\hline Art. 4 & 410 & Art. 2(4) & 397 \\
\hline Arts 5-12 & 391 & Art. 2(6) & 406 \\
\hline Art. 5 & 410 & Art. 2(7) & 406 \\
\hline Art. 6 & 410 & Art. 2(8) & 406 \\
\hline Art. 7 & 393,411 & Art. 2(9) & 406 \\
\hline Art. 8 & 393,411 & Art. 2(11) & 406 \\
\hline Art. 11 & 411 & Arts $3-5$ & $391,393,410$ \\
\hline
\end{tabular}




\begin{tabular}{|c|c|c|c|}
\hline Art. 3 & $391-4,414,421$ & Art. 13(2) & 415 \\
\hline Art. 3(1)(a) & 391, 392 & Art. 14 & 415,416 \\
\hline Art. 3(1)(a)(i) & 391 & Art. 15 & 416 \\
\hline Art. 3(1)(a)(ii) & 391 & Art. $15(1)$ & 416 \\
\hline Art. 3(1)(a)(iii) & 391 & Art. 15(2) & 416 \\
\hline Art. 3(1)(a)(iv) & 391 & Art. 15(3) & 416 \\
\hline Art. 3(1)(a)(v) & 391 & Art. 15(4) & 416 \\
\hline Art. 3(1)(a)(vi) & 391-3 & Art. 15(5) & 416,417 \\
\hline Art. 3(1)(b) & 391-3 & Art. 15(6) & 416 \\
\hline Art. 3(2) & 391, 392 & Arts $16-20$ & $391,395,410$ \\
\hline Art. 4 & $392,393,421$ & Art. 16 & 417 \\
\hline Art. 5 & 392,393 & Art. 17 & 395,417 \\
\hline Art. 6 & 391, 393-5, 411 & Art. 18 & 395,417 \\
\hline Art. 7 & 391, 393-5, 411 & Art. 19 & 395,417 \\
\hline Arts $8-15$ & 410,415 & Art. 20 & $396,415,417$ \\
\hline Art. 8 & 424 & Art. 20(1) & 417 \\
\hline Art. 8(1) & 412 & Art. 20(2) & 397,417 \\
\hline Art. 8(2) & 412 & Arts $21-52$ & 424 \\
\hline Art. 9 & 412 & Art. 21 & 398,425 \\
\hline \multirow[t]{2}{*}{ Art. 10} & $412,421,422$, & Art. 21(1) & 397 \\
\hline & 424 & Art. 21(2) & 397 \\
\hline Art. 10(a) & 421 & Art. 21(3) & 398 \\
\hline Art. 10(b) & 421,422 & Art. 21(4) & 397 \\
\hline Art. 11 & $412,422,424$ & Art. 22 & 398 \\
\hline Art. 11(1) & 422 & Art. 22(a) & 398 \\
\hline Art. 11(2) & 422 & Art. 22(b) & 399 \\
\hline Art. 11(3) & 422 & Art. 22(c) & 399 \\
\hline Art. 11(4) & 423 & Art. 22(d) & 399 \\
\hline Art. 11(5) & 423 & Arts $23-27$ & 398,426 \\
\hline Art. 11(6) & 423,424 & Art. 23 & 426 \\
\hline Art. 11(7) & $422-4$ & Art. 23(a) & 426 \\
\hline Art. 11(8) & $423,424,428$ & Art. 23(b) & 427 \\
\hline \multirow[t]{2}{*}{ Art. 12} & $397,412,414$, & Art. 23(c) & 426 \\
\hline & 415 & Art. 23(d) & 427 \\
\hline Art. 12(1) & 414 & Art. 23(e) & 427 \\
\hline Art. 12(2)(a) & 414 & Art. 23(f) & 427 \\
\hline Art. 12(2)(b) & 414 & Art. 23(g) & 427 \\
\hline Art. 12(2)(c) & 414 & Art. 24 & 398,426 \\
\hline Art. 12(3) & 415 & Art. 25 & 398 \\
\hline Art. 12(4) & 414 & Art. 26 & 398,426 \\
\hline Art. 13 & 412,415 & Art. 27 & 397 \\
\hline Art. 13(1) & 403,415 & Arts 28-39 & 398,425 \\
\hline
\end{tabular}




\begin{tabular}{|c|c|c|c|}
\hline Art. 28 & 426 & Arts 79-94 & 153 \\
\hline Art. 31(3) & 426 & Art. 80 & 153 \\
\hline Art. 36(2) & 398 & Art. 81 & 153 \\
\hline Arts $40-45$ & 428 & Art. 81(c) & 153 \\
\hline Art. 40(1)(b) & 428 & Art. $81(d)$ & 153 \\
\hline Art. 40(2) & 428 & Arts $84-7$ & 153 \\
\hline Art. 41 & 428,429 & Art. 95 & 153 \\
\hline Art. 41(1) & 428 & Euro Regulation & \\
\hline Art. 41(2) & 428,429 & $1103 / 97$ & 121 \\
\hline Art. 41(3) & 428 & \multicolumn{2}{|c|}{ Insolvency Regulation } \\
\hline Art. 42 & $387,428,429$ & $1346 / 2000$ & $28,29,214,439$ \\
\hline Art. 42(1) & 428 & Art. 1(1) & 29,440 \\
\hline Art. 42(2) & 428,429 & Art. 1(2) & 29,441 \\
\hline Art. 43 & 429 & Art. 2 & 29 \\
\hline Art. 44 & 429 & Art. 2(a) & 440 \\
\hline Art. 45 & 429 & Art. 2(d) & 441 \\
\hline Art. 46 & 425 & Art. 2(g) & 452 \\
\hline Art. 47(1) & 425 & Art. 2(h) & 451 \\
\hline Art. 47(2) & 425,429 & Art. 3 & 442,446 \\
\hline Art. 48 & 425 & Art. 3(1) & 443,444 \\
\hline Art. 49 & 387,425 & Art. 3(2) & $451,452,455$ \\
\hline Art. 56 & 427 & Art. 3(3) & 451 \\
\hline Art. 57(3) & 421 & Art. 3(4) & 455 \\
\hline Art. 59 & 398 & Art. 4 & $447,456,457$ \\
\hline Art. 60 & 386,408 & & 462 \\
\hline Art. 61 & 408,416 & Art. 4(2) & 456,457 \\
\hline Art. 62 & 408 & Art. $4(2)(d)$ & 460 \\
\hline \multirow[t]{3}{*}{ Art. 64} & $8,383,387$, & Art. 4(2)(e) & 461 \\
\hline & $388,398,403$, & Art. 4(2)(f) & 459 \\
\hline & 407 & Art. $4(2)(\mathrm{m})$ & 462 \\
\hline Art. 64(1) & 389,407 & Arts $5-15$ & $447,452,457$ \\
\hline Art. 64(2) & $388,389,407$ & & 462 \\
\hline Art. 64(3) & 390 & Art. 5 & $449,457,458$, \\
\hline Art. 64(4) & 390 & & 461 \\
\hline Art. 66 & 392 & Art. 5(1) & 458 \\
\hline Art. 66(a)-(b) & 392 & Art. 5(2) & 458 \\
\hline Art. 71 & $383,388,403$ & Art. 5(4) & 459 \\
\hline Art. 72 & $8,383,387,403$ & Art. 6 & 458,460 \\
\hline esign Regulation & & Art. 6(1) & 460 \\
\hline $6 / 2002$ & $41,97,153,366$ & Art. 6(2) & 460 \\
\hline Art. 52 & 153 & Art. 7 & 449, 457-9 \\
\hline Arts 79-83 & 97 & Art. 7(1) & 459 \\
\hline
\end{tabular}




\begin{tabular}{|c|c|c|c|}
\hline Art. 7(2) & 459 & Art. 35 & 453 \\
\hline Art. 7(3) & 459 & Art. 36 & 456 \\
\hline Art. 8 & $458,459,461$ & Art. 37 & 456 \\
\hline Art. 9 & 458,460 & Art. 38 & 446 \\
\hline Art. 10 & 458,461 & Arts 39-42 & 454 \\
\hline Art. 11 & 458,459 & Art. 39 & 455 \\
\hline Art. 12 & 452 & Art. 40 & 452 \\
\hline Art. 13 & 458,462 & Art. 41 & 452 \\
\hline Art. 14 & 458,459 & Art. 42 & 452 \\
\hline Art. 15 & $458-60$ & Art. 43 & 443 \\
\hline Art. 16 & $442,446,450$ & Art. 44 & 443 \\
\hline Art. 16(1) & $446-8,452,456$ & Art. 47 & 439 \\
\hline Art. 17(1) & $446,447,451$ & \multicolumn{2}{|c|}{ Plant Variety Regulation } \\
\hline Art. $17(2)$ & 452,456 & $2100 / 94$ & $41,97,366$ \\
\hline Art. 18(1) & 447,448 & Art. 20 & 151 \\
\hline Art. 18(2) & 452,456 & Art. 21 & 151 \\
\hline Art. 18(3) & 449 & Arts $94-100$ & 152 \\
\hline Art. 19 & 448 & Art. 101 & 97 \\
\hline Art. 20 & 449 & Art. 101(1) & 152 \\
\hline Art. 20(1) & 449,457 & Art. 101(2) & 152 \\
\hline Art. 20(2) & 449,457 & Art. 101(3) & 152 \\
\hline Arts $21-23$ & 447 & Art. 102 & 97 \\
\hline Art. 21 & 450 & Art. 102(3) & 152 \\
\hline Art. 24 & 450 & Art. 105 & 151 \\
\hline Art. 24(1) & 450 & Art. 106(2) & 152 \\
\hline Art. 24(2) & 450 & \multicolumn{2}{|c|}{ Service of Documents Regulation } \\
\hline \multirow[t]{2}{*}{ Art. 25} & $30,239,442$, & $1348 / 2000$ & 10 \\
\hline & 450 & Art. 19 & 55,230 \\
\hline Art. 25(3) & 451 & \multicolumn{2}{|c|}{ Social Security Regulation } \\
\hline \multirow[t]{2}{*}{ Art. 26} & $30,447,448$, & $1408 / 71$ & 31 \\
\hline & 450 & \multicolumn{2}{|c|}{ Taking of Evidence Regulation } \\
\hline Art. 27 & 451,452 & $1206 / 2001$ & 10,405 \\
\hline Art. 28 & 452,456 & \multicolumn{2}{|c|}{ Trade Mark Regulation } \\
\hline Art. 29 & 451 & \multirow[t]{2}{*}{$40 / 94$} & $41,97,148$ \\
\hline Art. 31 & 452 & & 153,366 \\
\hline Art. 32 & 454 & Art. 56 & 148 \\
\hline Art. 32(1) & 452 & Arts 57-63 & 148 \\
\hline Art. 32(2) & 452 & Arts $90-104$ & 97,148 \\
\hline Art. 32(3) & 453 & Art. 90 & 149 \\
\hline Art. 33 & 453 & Art. 90(1) & 150 \\
\hline Art. 34 & 453 & Art. 90(2)(a) & 148,150 \\
\hline Art. 34(2) & 452 & Art. 90(2)(b) & 149 \\
\hline
\end{tabular}




\begin{tabular}{|c|c|c|c|}
\hline Art. $90(2)(\mathrm{c})$ & 149 & Art. 4(2) & 251 \\
\hline Art. 91 & 148 & Art. 4(3) & 252 \\
\hline Art. 92 & 148 & Art. 4(6) & 253 \\
\hline Art. 92(d) & 148 & Art. 4(7) & 252 \\
\hline Art. 93 & 148 & Arts 5-11 & 253 \\
\hline Art. 93(1)-(3) & 148 & Art. 5 & 253 \\
\hline Art. 93(1) & 149 & Art. 6(1) & 253 \\
\hline Art. 93(2) & 149 & Art. 6(1)(a) & 253 \\
\hline Art. 93(3) & 149 & Art. 6(1)(b) & 253 \\
\hline Art. 93(4) & 149 & Art. 6(1)(c) & 254 \\
\hline Art. 93(5) & 149 & Art. $6(1)(d)$ & 254 \\
\hline Art. 94 & 148 & Art. 6(2) & 253 \\
\hline Art. 94(1) & 148 & Art. 6(3) & 253,254 \\
\hline Art. 94(2) & 149 & Art. 7 & 253 \\
\hline Art. 96 & 148 & Art. 8 & 253 \\
\hline Art. 96(2) & 148 & Art. 9 & 253 \\
\hline Art. 96(7) & 150 & Art. 10 & 258 \\
\hline Art. 99(1) & 150 & Art. 10(1) & 254 \\
\hline Art. 99(2) & 150 & Art. 10(2) & 254 \\
\hline Art. 100 & 149 & Art. 10(3) & 254 \\
\hline Art. $100(1)$ & 149 & Art. 10(4) & 254 \\
\hline Art. $100(2)$ & 149,150 & Art. 11 & 253 \\
\hline Art. 100(3) & 150 & Arts $12-19$ & 254 \\
\hline Art. 102 & 150 & Art. 12 & 254 \\
\hline Art. 102(1) & 150 & Art. 12(2) & 254 \\
\hline Art. 102(2) & 150 & Arts $13-15$ & 255,256 \\
\hline Art. 105 & 150,153 & Art. 13 & 255,256 \\
\hline Art. 105(1) & 151 & Art. 14 & $255-7$ \\
\hline Art. 105(1)(a) & 151 & Art. 14(2) & 255 \\
\hline Art. 105(1)(b) & 151 & Art. 14(3) & 255 \\
\hline Art. 105(2) & 151 & Art. 15 & 256 \\
\hline Art. 105(3) & 151 & Art. 16 & 256 \\
\hline Art. 105(4) & 151 & Art. 17 & 256 \\
\hline ncontested Claim & Regulation & Art. 18 & 256 \\
\hline $805 / 2004$ & $7,213,250$ & Art. 18(1) & 256 \\
\hline Art. 1 & 250 & Art. 18(2) & 257 \\
\hline Art. 2(1) & 251 & Art. 19 & 257,258 \\
\hline Art. 2(2) & 251 & Art. 19(1)(a) & 257 \\
\hline Art. 2(3) & 250 & Art. 19(1)(b) & 257 \\
\hline Art. 3 & 251 & Art. 19(2) & 257 \\
\hline Art. 3(1) & $251,253,254$ & Arts $20-23$ & 257 \\
\hline Art. 4(1) & 252 & Art. 20(1) & 257 \\
\hline
\end{tabular}




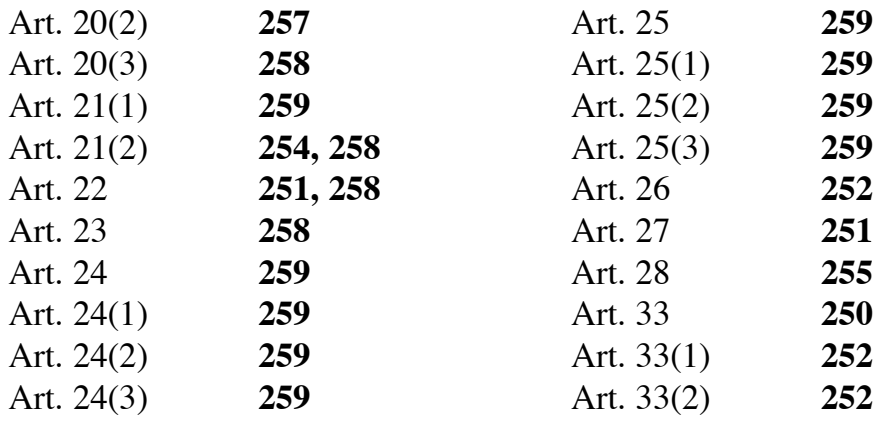

